\title{
Predicting the viability of archaic human hybrids using a mitochondrial proxy
}

Richard Allen ${ }^{1 *}$, Hannah Ryan ${ }^{1 *}$, Brian W. Davis ${ }^{2}$, Charlotte King ${ }^{10,3}$, Laurent Frantz $^{1,8}$, Ross Barnett ${ }^{1}$, Anna Linderholm ${ }^{1,6}$, Liisa Loog ${ }^{1,7}$, James Haile ${ }^{1}$, Ophélie Lebrasseur $^{1}$, Mark White ${ }^{3}$, Andrew C. Kitchener ${ }^{5,9}$, William J. Murphy ${ }^{4}$, Greger Larson $^{1}$

${ }^{1}$ Palaeogenomics \& Bio-Archaeology Research Network, Research Laboratory for Archaeology and the History of Art, University of Oxford, Oxford OX1 3QY, UK

${ }^{2}$ Cancer Genetics Branch, National Human Genome Research Institute, NIH, Bethesda, Maryland 20892, USA

${ }^{3}$ Department of Archaeology, Durham University, Science Site, Durham, UK DH1 3LE

${ }^{4}$ Veterinary Integrative Biosciences, Texas A\&M University, College Station TX 77843 USA

${ }^{5}$ Department of Natural Sciences, National Museums Scotland, Chambers Street, Edinburgh EH1 IJF, UK

${ }^{6}$ Department of Anthropology, Texas A\&M University, College Station, TX 778434352, USA

${ }^{7}$ Department of Zoology, University of Cambridge, Downing Street, Cambridge CB2 3EJ, United Kingdom

${ }^{8}$ School of Biological and Chemical Sciences, Queen Mary University of London, Mile End Road, London E1 4NS, UK

${ }^{9}$ Institute of Geography, School of Geosciences, University of Edinburgh, Drummond Street, Edinburgh EH9 3PX, UK

${ }^{10}$ Department of Anatomy, University of Otago, Great King Street, Dunedin 9016, NZ

* These authors contributed equally to this work.

Corresponding author: Greger Larson, greger.larson@arch.ox.ac.uk 
41

\section{Abstract}

Ancient DNA evidence has confirmed hybridization between humans and Neanderthals and revealed a complex pattern of admixture between hominin lineages. Many segments of the modern human genome are devoid of Neanderthal ancestry, however, and this non-random distribution has raised questions regarding the frequency and success of hybridisation between ancient human lineages. Here, we examine the hypothesis that hominin hybrid offspring suffered a reduction in fertility by comparing patterns of sequence divergence of mitochondrial and nuclear DNA from numerous hybridising pairs of mammals. Our results reveal a threshold separating species pairs whose divergence values fall within two categories: those whose hybrid offspring can successfully reproduce without backcrossing with their parent species, and those whose hybrid offspring cannot. Using this framework, we predict that the potential hybrid offspring of Neanderthals, Denisovans, the ancient individuals from the Sima de los Huesos and anatomically modern humans would not have suffered a reduction in fertility.

\section{Introduction}

Though numerous interspecies hybrids have been recorded, predicting their viability and relative fertility has proved difficult. For example, the absence of Neanderthal mitochondrial genomes in the modern human population led some to suggest that anatomically modern humans (AMH) and Neanderthals did not hybridise ${ }^{1-3}$. Recent analyses of whole genome sequences derived from ancient individuals, however, have demonstrated that archaic hominins including Neanderthals and Denisovans did in fact hybridise with $\mathrm{AMH}^{4-6}$. For many anthropologists, this result corroborated previous conclusions (based on morphological grounds) that Neanderthals and AMH could have produced fertile offspring $\left(e . g .{ }^{7}\right)$.

A recent paper, however, conflated the observation of genetic deserts devoid of Neanderthal ancestry on modern human autosomes, and especially the $\mathrm{X}$ chromosome, with reproductive incompatibilities between humans and Neanderthal ${ }^{8}$. Though this study also suggested that demographic factors likely played a role in the formation of these deserts, two studies ${ }^{9,10}$ concluded that most selection against Neanderthal DNA in genic regions was not due to reproductive incompatibilities, but instead was the result of the Neanderthals' high genetic load. In addition, the initially 
75 high proportion of Neanderthal admixture ${ }^{11}$ suggests that early male and female

76 hybrids of AMH and Neanderthals were normally fertile, and that any Neanderthal variants that may have interfered with fertility within an AMH genetic background were likely confined to a few genetic loci. Lastly, multiple factors including ecological drivers can lead to genomic deficits of introgression within hybrid offspring that are otherwise fertile ${ }^{12}$, suggesting the existence of genetic deserts may not imply reproductive incompatibility.

More generally, assessing whether any two species are capable of producing viable or fertile offspring is difficult in the absence of nuclear genomic data, captive experiments or field data. In addition, there has been no robust, quantitative biological measure of the expected fitness of hybrid offspring, and thus no rigorous method to ascertain the specific viability of archaic human hybrids, or the potential for hybridisation between more ancient hominin lineages. Determining whether distinct hominin lineages were capable of producing fertile offspring is important not only for understanding the patterns of hybrid ancestry within modern humans, but also for ascertaining the degree to which reproductive isolation (as a result of either cultural or biological phenomena) existed between $\mathrm{AMH}$ and other hominin lineages.

Though numerous studies have speculated about the potential for hominin lineages to produce viable hybrids, few have made use of the numerous pairs of mammalian species known to produce hybrid offspring along a continuum of male (and to a lesser extent, female) fertility. Based on the qualitative correlation that the fertility of hybrids generally decreases with divergence time, two recent studies ${ }^{13,14}$ speculated that, given their relatively recent temporal divergence, $\mathrm{AMH}$ and Neanderthals could

100 have retained the ability to produce fertile offspring. These studies could not rule out

101 the fact that the male hybrids may have possessed lower fertility as a result of offspring, it is the heterogametic sex.

105 In order to obtain a quantitative measure of whether two lineages can interbreed, we

106 developed a framework based on the correlation between mitochondrial genetic

107 distance between mammalian species pairs, and the manifestation of hybrid male

108 sterility. Some previous studies have questioned this approach and stated that 
109 measures of species divergence are not necessarily reliable predictors of hybrid

110 sterility $^{15,16}$. Other studies have reported that genetic divergence values do correlate

111 with species boundaries ${ }^{17}$ and time to speciation. A more specific recent study of

112 damselflies demonstrated a strong correlation between the genetic distances

113 separating two species and their relative reproductive isolation ${ }^{18}$.

114

115 Hybrid fertility exists along a continuum. For the sake of simplicity and ease of

116 distinction, we first explicitly defined two dichotomous categories amongst hybrid

117 offspring. Category 1 contains eight terrestrial mammalian species pairs that are

118 capable of producing F1 offspring that can reproduce without backcrossing with a

119 parent species (even if there are observed asymmetries in gene flow and variation in

120 male fertility amongst the hybrids) (Table S1). Category 2 consists of nine pairs of

121 species that can produce viable F1 offspring, but these hybrids either cannot

122 reproduce without backcrossing with a parent species, or are completely infertile

123 (Table S1). In order to determine the categorical assignment of each species pair, we

124 relied upon empirical evidence derived from experimental studies of F1 hybrid

125 fertility (Table S1). We also compiled 22 additional species pairs that are known to

126 produce viable offspring, but for which there was insufficient evidence to confidently

127 assign them into either category (Table S2, Fig. S2).

128

129 We then aligned sequences of both the cytochrome b gene $(C Y T B)$ and mitochondrial

130 genomes (excluding the control region) from multiple individuals per species and

131 confirmed (by matching the phylogenies to nuclear species trees) that the selected

132 mitochondrial sequences for each taxon pair were neither mislabelled, nor nuclear

133 copies of mitochondrial genes, nor derived from hybrid populations. From the

134 sequence alignments we calculated average pairwise genetic distances between each

135 species pair using both Hamming (raw) distances and evolutionary models. In

136 addition, we calculated genetic distances between ten primate species pairs using four

137 nuclear loci (CHRNA1, GHR, ZFX, ZFY) ${ }^{19}$ (Fig. S3).

\section{Results and Discussion}

141 The calculated divergence estimates using CYTB revealed clear thresholds

142 distinguishing the two categories (Fig 1, Fig. S1). More specifically, none of the eight 
143 Category 1 pairs that were capable of producing fully fertile offspring possessed

144 CYTB sequences with raw average pairwise divergence values greater than $7.60 \%$.

145 Other than two vole species that possessed a divergence value of $7.54 \%$, all other

146 Category 2 pairs possessed divergence values greater than $7.73 \%$. The existence of a

147 genetic distance threshold also held true for the mitogenomes (Fig. S3). In addition,

148 the male and female hybrid offspring of the two most divergent pairs were shown to

149 be completely infertile corroborating previous studies ${ }^{20-22}$ showing that along the

150 continuum of speciation, infertility evolves prior to inviability. Lastly, a Mann-

151 Whitney $U$ test showed significantly lower genetic divergence values of species pairs

152 in Category 1 relative to those in Category $2(\mathrm{p}<0.0001)$.

153

154 Importantly, the two categories of fertility defined here are not strictly linked with gene flow. For instance, though both male and female hybrid offspring of all species pairs in Category 1 can reproduce without requiring a backcross with a parent species gene flow asymmetries have been demonstrated in virtually all Category 1 pairs including house mice (Mus musculus musculus x Mus musculus domesticus) ${ }^{23}$, and brown (Ursus arctos) and polar bears (Ursus maritimus) ${ }^{12}$ (Table S1). In addition, gene flow has also been demonstrated between Category 2 species (including Mus musculus and M. spretus $^{24}$ ) whose F1 hybrid offspring cannot produce viable F2s. Since both fertility and the potential for gene flow vary along a continuum, it is interesting to note that the divergence values associated with the two fertility categories defined here do not overlap more substantially. As a result, our results suggest that pairwise mitochondrial genetic distance values can be used to make predictions of male hybrid sterility, at least in terrestrial mammals.

Though the lack of available sequences for nuclear loci (relative to mitochondrial loci) limited our ability to test whether nuclear genes produced the same general pattern as the mitochondrial loci across all our species pairs, we were able to identify four nuclear genes: Zinc finger Y-chromosomal protein (ZFY), Zinc finger Xchromosomal protein (ZFX), Growth Hormone Receptor (GHR), Cholinergic Receptor Nicotinic Alpha (CHRNA1), that had been sequenced in 10 of the primate pairs (Fig. S3) that were known to produce viable hybrid offspring ${ }^{19}$. We generated pairwise distances for each of these genes using the same methodology employed in the mitochondrial analyses. We then assigned each species pair to Category 1 or 
177 Category 2 based upon their CYTB divergence values within the original framework.

178 In each case, though the order of the taxa based upon pairwise divergence values

179 varied relative to the pattern generated using CYTB (owing to the significantly smaller

180 divergence values in nuclear loci), for each locus the limited overlap in divergence

181 values associated with the two categories was consistent with the mitochondrial

182 assessment (Fig. S3).

183

184 In order to further substantiate both this correlation and the robustness of $C Y T B$ as a

185 proxy for hybrid fertility, we tested the utility of this system for predicting fertility in

186 a well-known hybrid system. To create hybrid pets, cat breeders have crossed

187 domestic cats (Felis catus) with three species of wild felid: Jungle cats (F. chaus),

188 Leopard cats (Prionailurus bengalensis), and Servals (Leptailurus serval) ${ }^{25}$. In all

189 cases, the first generation male hybrids are sterile. To regain fertility while

190 maintaining some wild cat characteristics, breeders must backcross the F1 female

191 offspring with male domestic cats to establish a breeding population of pets ${ }^{25}$. Given

192 that backcrossing is required for all three crosses, our framework would firstly predict

193 that the $C Y T B$ distances between all three pairs should be close to or greater than

$194 \sim 7.5 \%$ and that they should all fall into the Category 2 range. Secondly, the pairs with

195 greater genetic distances should require more numerous backcrosses with domestic

196 cats (halving the wild cat ancestry with each subsequent generation) before full

197 fertility is restored and a breeding pet population is established.

199 Both of these predictions are borne out by the data (Fig 1, Table S3). All three pairs

200 possess $C Y T B$ distances greater than or equal to $7.54 \%$ and the increasing molecular

201 distances between the pairs correlate with an increase in the number of required

202 backcross generations to regain fertility. Specifically, distances between Jungle cats,

203 Leopard cats, Servals and domestic cats (7.54\%, 10.94\%, and $11.28 \%$ respectively)

204 are consistent with both the observed minimum (2, 3, and 4 respectively) and average

$205(3,4$, and 5, respectively) number of backcrosses with domestic cats required for

206 hybrid males to acquire fertility ${ }^{25}$. These results are also consistent with an early

207 hybrid experiment using guinea pigs in which hybrids between Cavia fulgida and $C$.

208 porcellus (8.02\% CYTB divergence) were able to regain male fertility after the third

209 generation of backcrosses ${ }^{26}$ (Table S1). 
211 Recent accidental hybrids in zoos also confirm the predictive power of this proxy. In

212 2006, the Copenhagen Zoo placed a domestic sow (Sus scrofa domesticus) in a pen

213 with a male babirusa (Babyrousa celebensis) with the expectation that the two species

214 were sufficiently evolutionarily divergent that they would be incapable of producing

215 offspring. The CYTB divergence between the two species (12.89\%), however, falls

216 within the Category 2 range. Months later, five infertile piglets were born and though

217 two died from maternally induced trauma, the other three (two males, and one

218 female), all survived ${ }^{27}$ (Table S1, Fig. S1). Historically, zoos have often accidentally

219 produced hybrids offspring between distantly related species (Table S2), though the

220 relative fertility of the F1s was rarely established.

221

222 Having approximated the $C Y T B$ threshold values within the framework, we then 223 predicted the relative fertility of hybrids between pairs of other hominin lineages. To 224 do so, we calculated the average pairwise divergence in $C Y T B$ sequences between $225 \mathrm{AMH}$ and three extinct hominin lineages: Neanderthals, Denisovans, and the ancient 226 population from the Sima de los Huesos cave in Spain ${ }^{28}$. To avoid overestimating the 227 genetic divergence that results from comparing modern and extinct populations, we 228 also generated distance values using the CYTB sequences derived solely from ancient 229 AMH found in archaeological contexts.

231 The divergence values for each pairing between three Homo groups (Sima de los 232 Huesos, Neanderthals, and AMH) all possess divergence values at the bottom of the 233 Category 1 range (Fig 1, Fig. S1, Table S1). Interestingly, the divergence values of 234 Denisovan-Neanderthal and Denisovan-AMH are the largest of the Homo pairings, 235 and are consistent with the suggestion that Denisovans possess a mitochondrial 236 lineage that may have introgressed from another source population ${ }^{29}$.

238 In order to assess hybrid fertility within the hominin lineage more deeply, we 239 calculated the pairwise $C Y T B$ divergence between humans and our closest living 240 relatives: Chimpanzees (Pan troglodytes) and bonobos (P. paniscus). Female 241 chimpanzees inseminated with human sperm during a Soviet experiment in the 1920s 242 failed to produce any offspring, and the reverse experiment did not progress beyond 243 the planning stage ${ }^{30}$. Recent molecular clock assessments have suggested that AMH 244 and chimps diverged $\sim 5-6 \mathrm{Mya}^{31}$, well beyond both the two million year threshold 
245 purported by other studies as the upper limit to hybrid fertility ${ }^{14}$, and the average time 246 to speciation $^{32}$.

248 Interestingly, our analysis places the divergence values between AMH and chimps, 249 and AMH and bonobos within Category 2 (Fig 1, Fig S1). Six other species pairs

250 (including two of the wild cat and domestic cat pairs) possess divergence values

251 equivalent to or greater than AMH, chimps, and bonobos (Table S1, Fig. S1). In all

252 six cases, however, each of the pairs possesses an identical number of chromosomes.

253 Though karyotype is not a strict barrier to the production of hybrids (Table S1), AMH

254 possess two fewer chromosomes than chimps and bonobos (Table S1).

255

256 Conclusions

257 When placed within the context of other mammalian species, ancient hominin

258 lineages were likely not sufficiently divergent from each other to expect a biological

259 impediment to the generation of fertile offspring. Although some studies have inferred

260 a low level of introgression ${ }^{33}$, the relative ease of hybridisation between hominin

261 lineages is demonstrated by the fact that archaic populations interbred with AMH on

262 at least four occasions ${ }^{34}$. The absence of a biological barrier shifts the focus toward

263 cultural considerations and demographic factors to explain the lack of Neanderthal

264 and Denisovan genomic contribution on the AMH X chromosome, and the lack of

265 mitochondrial DNA from either species within AMH. Palaeoanthropologists disagree

266 as to what differences in culture, behaviour and cognition existed between AMH and

267 Neanderthals (e.g. ${ }^{35,36}$ ). Though these factors may have altered the sexual symmetry

268 of the hybrids, they appear not to have precluded introgression in either direction ${ }^{37}$.

270 This study demonstrates the power of genetic distances as a proxy to predict the 271 relative sterility of hybrids resulting from matings between mammalian species.

272 Hybrid sterility is both fluid and governed by numerous mechanisms at multiple 273 levels of biological organization. In addition, gene flow between species whose 274 hybrids require backcrossing is possible (Table S1), as are asymmetries in gene flow 275 in species pairs whose F1 offspring can produce fertile F2s. We do not claim that 276 CYTB plays a causative role in hybrid fertility (though a recent study proposed that 277 speciation may be mediated by mitonuclear interactions ${ }^{38}$ ), and the use of genetic 278 divergence values as a proxy cannot be perfectly predictive. For example, under the 
279 Dobzhansky-Müller model, incompatibility can arise from as few as two mutations in 280 each of the admixing populations irrespective of time since divergence, meaning that 281 it would be possible for closely related populations to be incapable of generating 282 fertile hybrids ${ }^{39}$, though no such examples have yet been described.

283 The value of any proxy, however, is determined by both its predictive power and the 284 ease of generating the proxy data. Publicly available mtDNA sequences from 285 thousands of mammalian taxa already exist and calculating pairwise divergence 286 values is inexpensive, simple and fast. As a result, CYTB specifically (and genomic 287 distances more generally) have substantial value as a means to predict the potential 288 for any two mammalian species to produce viable offspring, and the relative degree of 289 the hybrid's sterility. The discovery of additional extinct hominin populations that 290 survived into the last 250,000 years, including $H$. floresiensis and $H$. naledi, has 291 raised interest in understanding the limits to fertility and hybridization between 292 extinct and extant Homo spp. If and when mitochondrial genomes from these samples 293 can be obtained, the approach described here may provide an answer, even if nuclear 294 genomic data are not obtainable.

296 Lastly, establishing which species pairs violate the predictions of the framework will 297 lead to a better understanding of the process of speciation and the biological and 298 sociocultural mechanisms responsible for hybrid sterility. Our framework can also be 299 applied more generally in contexts such as conservation biology, where predicting the 300 consequences of biological hybridization is key to policy considerations.

\section{Materials and Methods}

303

\section{Assessment of Hybrid Fertility and Rationale of Assignment into Categories}

305 In order to ascertain if there was a relationship between genetic divergence and the

306 fertility of hybrid offspring between species, we first collected published examples of

307 species pairs that were capable of producing live offspring. We then split the hybrid

308 pairings into two categories. Into Category 1 we placed eight species pairs that are

309 capable of producing fertile F1 offspring of both sexes that can reproduce without 310 backcrossing with a parent species. Though within these examples there have been 311 observed asymmetries in gene flow and variation in fertility amongst the hybrids, 
312 none of these examples requires (one or more generations of) backcrossing with a

313 parent species for their offspring to regain fertility. More specifically, for an example

314 to be listed in this category, we required evidence of fertility in both male and female

315 F1 hybrids. The evidence and rationale for placing each of these pairs into Category 1

316 is listed in Table S1.

318 The hybrid offspring of all of nine pairs of species in Category 2 either require one or

319 more generations of female hybrids backcrossing with the male of a parent species to

320 produce fertile offspring, or are completely infertile. For these pairs, we obtained

321 evidence demonstrating no successful F2s from F1 hybrid couplings, an inability to

322 produce offspring other than by backcrossing to a parent species, or other biological

323 assessments (including a histological assessment of the testis from the hybrid males)

324 that demonstrated complete infertility (Table S1).

326 For instance, in the case of hybrids between lions (Panthera leo) and tigers (P. tigris),

327 there are numerous anecdotal reports regarding hybrid male sterility in ligers (male

328 lion $\mathrm{x}$ female tiger) and the backcross hybrid offspring derived from females (e.g. ${ }^{40}$.

329 Here, we provide histological evidence of sterility based on H\&E stained testes from

330 an adult male liger (Fig. S4 panels a and b), and an adult male tiliger (male tiger $\mathrm{x}$

331 female liger; panels $\mathrm{c}$ and d). Testes show clear seminiferous tubule degeneration,

332 lined only with Sertoli cells in the liger, and tubule degeneration with germ cell arrest

333 in the tiliger (Fig. S4 panels c, d).

335 Many additional live hybrid offspring have been reported in the literature than are

336 included in Fig. 1 or in Fig. S1. The relative fertility of the hybrid offspring, however,

337 has not been adequately assessed and we were therefore unable to confidently place

338 them into either category. The framework and threshold values depicted in Fig. 1

339 allow us to predict the fertility of these offspring given the definitions described

340 above and their placement into Category 1 or 2 . These pairs are listed in Table S2 and

341 their relative positions are depicted in Fig. S2.

\section{Genetic Distance Calculation}

344 Both $C Y T B$ sequences and full mitogenomes (excluding the control region) of

345 multiple individuals of each species were collected from Genbank (Table S4) and 
346 aligned using Clustal Omega version 1.2.4 $4^{41}$. In order to ensure that none of the

347 sequences was either mislabelled or were nuclear copies of mitochondrial genes, we

348 constructed Neighbour-Joining trees using Geneious version $6.1 .8^{42}$ and removed all

349 individuals that did not fall into monophyletic clades of each species. We first used

350 pModelTest version $1.04^{43}$ to determine the best model for the alignment of each set

351 of sequences for both species. We then calculated pairwise distances between each

352 species pair using RAXML version $8^{44}$ and FASTTree version $2.1^{45}$. We also

353 generated raw distance values (i.e. the proportion of sites that differ between each pair

354 of sequences) using the Hamming distance method which sums the number of

355 basepair differences, regardless of whether they are transitions or transversions, and

356 divides that number by the sequence length.

357

358 The distances were generated from the $C Y T B$ (and nuclear gene) alignments for each

359 set of species pairings in fasta file format using a python wrapper to wrap around the

360 programs RAXML, FastTree, pModelTest, and a custom python script written to test

361 Hamming distance of sequences making use of the distance version $0.1 .3^{46}$ and

362 Biopython version $1.68^{47}$ modules. The script was written for use in version 2.7 of the

363 Terminal application in an OSX environment, and the source code is included at the

364 end of this Supplementary Information. To generate the Hamming distance

365 calculations, the aligned sequences had to be the identical length (meaning all partial

366 sequences were rejected). In addition, gaps in the published sequences were ignored

367 across those loci and across each alignment even if a nucleotide was present at those

368 loci in other sequences to minimise the artificial inflation of the divergence value

369 differences.

370

371 Mann-Whitney test of statistical difference between CYTB distance in hybrid

372 categories

373 The statistical significance of observed differences in mitochondrial genetic distance

374 between groups with reduced hybrid compatibility and those with full compatibility,

375 was tested using the Mann-Whitney U test ( $\mathrm{p}=5.439 \mathrm{e}-06)$ implemented in the R-

376 software package(R Core Team $2013^{48}$ ).

\section{$378 \quad$ Figure Legends}




\section{$380 \quad$ Figure 1}

381 A depiction of the correlation between $C Y T B$ divergence between terrestrial

382 mammalian species pairs and the relative fertility of their hybrid offspring. In column

383 A, the green circles represent species capable of producing fully fertile F1 offspring

384 which can reproduce independently of their parent species (Category 1). Brown

385 circles represent species pairs whose F1 offspring either require backcrossing with a

386 parent species or are completely infertile (Category 2). The lighter green and brown

387 shaded regions represent the range of divergence values of the two categories and,

388 given their general lack of overlap, can be used to determine the relative fertility of

389 hybrid offspring. Column B depicts the divergence between three wild felid species

390 and domestic cats, as well as the minimum number of generations of backcrosses with

391 domestic cats before full fertility of the hybrid is restored. The white circles in

392 Column $\mathrm{C}$ depict the divergence between three ancient hominins and AMH, as well as

393 the distances between AMH and chimpanzees and bonobos (in Category 2). The

394 asterisks represent those pairs that include modern samples of AMH. The lack of an

395 asterisk signifies that only sequences derived from archaeological AMH were used to

396 compute the divergence values. All hominin pairs are positioned at the lowest (most

397 fertile) end of Category 1, suggesting that their hybrid offspring are likely to have

398 been fully fertile. Additional detail regarding the specific species pairs are listed in

399 Fig. S1 and Table S1.

400

401 Acknowledgments

402 We thank Simon Ho, Linda Maxson, Julie Wilson, Andrew Millard, John Hawks,

403 Tom Higham, Kelly Harris, Christian Capelli, Shyam Gopalakirshnan, Montgomery

404 Slatkin, Josh Schraiber, Sam Turvey, Janet Kelso, and Alfred Roca, for advice and

405 discussion. We also thank the Zoological Society and the Bartlet Society for their

406 assistance. GL was supported by the European Research Council (ERC-2013-StG

407 337574-UNDEAD) and the Natural Environment Research Council (NE/H005269/1

408 \& NE/K005243/1).

409

410

411

\section{References}


46726 Detlefsen, J. A. Genetic studies on a Cavy species cross. 205 (1914).

46827 Thomsen, P. D. et al. Meiotic studies in infertile domestic pig-babirusa hybrids. Cytogenetic and Genome Research 132, 124-128, doi:10.1159/000320421 (2011).

28 Meyer, M. et al. A mitochondrial genome sequence of a hominin from Sima de los Huesos. Nature 505, 403-406 (2014).

29 Meyer, M. et al. A High-Coverage Genome Sequence from an Archaic Denisovan Individual. Science 338, 222-226, doi:10.1126/science.1224344 (2012).

30 Etkind, A. Beyond eugenics: the forgotten scandal of hybridizing humans and apes. Studies in History and Philosophy of Science Part C: Studies in History and Philosophy of Biological and Biomedical Sciences 39, 205-210 (2008).

31 Scally, A. et al. Insights into hominid evolution from the gorilla genome sequence. Nature 483, 169-175 (2012).

32 Hedges, S. B., Marin, J., Suleski, M., Paymer, M. \& Kumar, S. Tree of life reveals clock-like speciation and diversification. Molecular biology and evolution, msv037 (2015).

33 Currat, M. \& Excoffier, L. Strong reproductive isolation between humans and Neanderthals inferred from observed patterns of introgression. Proceedings of the National Academy of Sciences 108, 15129-15134 (2011).

34 Vernot, B. et al. Excavating Neandertal and Denisovan DNA from the genomes of Melanesian individuals. Science 352, 235-239 (2016).

35 Klein, R. G. Whither the Neanderthals? Science 299, 1525-1527 (2003).

36 Nowell, A. Defining behavioral modernity in the context of Neandertal and anatomically modern human populations. Annual Review of Anthropology 39, 437452 (2010).

37 Kuhlwilm, M. et al. Ancient gene flow from early modern humans into Eastern Neanderthals. Nature 530, 429-433 (2016).

38 Hill, G. E. Mitonuclear coevolution as the genesis of speciation and the mitochondrial DNA barcode gap. Ecology and Evolution 6, 5831-5842 (2016).

39 Orr, H. A. \& Turelli, M. The evolution of postzygotic isolation: accumulating Dobzhansky-Muller incompatibilities. Evolution 55, 1085-1094, doi:10.1111/j.00143820.2001.tb00628.x (2001).

40 Gray, A. P. Mammalian hybrids. A check-list with bibliography. [Second edition]. Commonwealth Agricultural Bureaux Slough i-x, 1-262 (1972).

41 Sievers, F. et al. Fast, scalable generation of high-quality protein multiple sequence alignments using Clustal Omega. Molecular Systems Biology 7, 539-539, doi:10.1038/msb.2011.75 (2014).

42 Kearse, M. et al. Geneious Basic: An integrated and extendable desktop software platform for the organization and analysis of sequence data. Bioinformatics 28, 16471649, doi:10.1093/bioinformatics/bts199 (2012).

43 Serra, F. pModelTest. (GitHub, 2011).

44 Stamatakis, A. RAxML version 8: a tool for phylogenetic analysis and post-analysis of large phylogenies. Bioinformatics 30, 1312-1313, doi:10.1093/bioinformatics/btu033 (2014).

45 Price, M. N., Dehal, P. S. \& Arkin, A. P. FastTree 2--approximately maximumlikelihood trees for large alignments. PloS one 5, e9490, doi:10.1371/journal.pone.0009490 (2010).

46 Meyer, M. distance. (GitHub, 2013).

47 Cock, P. J. a. et al. Biopython: freely available Python tools for compu... [Bioinformatics. 2009] - PubMed result. Bioinformatics (Oxford, England) 25, 14221423, doi:10.1093/bioinformatics/btp163 (2009). 


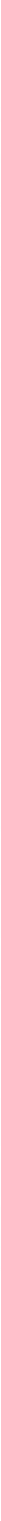

